\title{
STATISTICAL APPLICATIONS FOR EQUIVARIANT MATRICES
}

\author{
S. H. ALKARNI
}

(Received 24 November 1999)

\begin{abstract}
Solving linear system of equations $A \mathbf{x}=\mathbf{b}$ enters into many scientific applications. In this paper, we consider a special kind of linear systems, the matrix $A$ is an equivariant matrix with respect to a finite group of permutations. Examples of this kind are special Toeplitz matrices, circulant matrices, and others. The equivariance property of $A$ may be used to reduce the cost of computation for solving linear systems. We will show that the quadratic form is invariant with respect to a permutation matrix. This helps to know the multiplicity of eigenvalues of a matrix and yields corresponding eigenvectors at a low computational cost. Applications for such systems from the area of statistics will be presented. These include Fourier transforms on a symmetric group as part of statistical analysis of rankings in an election, spectral analysis in stationary processes, prediction of stationary processes and Yule-Walker equations and parameter estimation for autoregressive processes.
\end{abstract}

2000 Mathematics Subject Classification. Primary 65-XX, 65-04.

1. Introduction. Many problems in science and mathematics exhibit equivariant phenomena which can be exploited to achieve a significant cost reduction in their numerical treatment. Recent monographs $[16,17,21]$ have shown the efficiency of applying group theoretical methods in the study of various problems having symmetry properties. Allgower et al. [2, 4] presented some techniques for exploiting symmetry in the numerical treatment of linear integral equations, with emphasis on boundary integral methods in three dimensions. Georg and Tausch [15] introduced a user's guide for a software package to solve equivariant linear systems. Definitions for this subject are introduced first.

\subsection{Definitions}

\section{DEFINITION 1.1}

GROUP. An ordered pair $(\Gamma, o)$ is a group if $\Gamma$ is a set and $o$ is an operation $o: \Gamma \times \Gamma \rightarrow \Gamma$ such that

(a) for any $\gamma_{1}, \gamma_{2} \in S \Rightarrow \gamma_{1} o \gamma_{2} \in \Gamma$,

(b) there exists $e \in \Gamma$ such that $e o \gamma=\gamma$,

(c) $\left(\gamma_{1} o \gamma_{2}\right) o \gamma_{3}=\gamma_{1} o\left(\gamma_{2} o \gamma_{3}\right)$,

(d) there exists $\gamma^{-1} \in \Gamma$ such that $\gamma o \gamma^{-1}=e$.

\section{DEFINITION 1.2}

EQUIVARIANT MATRICES. An $n \times n$ matrix $A$ is equivariant with respect to a finite group $G$ of permutations if, for every $s \in G$, 


$$
A \Pi(s)=\Pi(s) A,
$$

where $\Pi(s)$ is the permutation matrix for $s \in G$. For more information on group theoretical methods and their applications, see Fässler and Stiefel [14].

Note that (1.1) is equivalent to

$$
A=\Pi^{-1}(s) A \Pi(s) .
$$

Note also since $\Pi=\left(e_{\pi(1)}, e_{\pi(2)}, \ldots, e_{\pi(n)}\right)$ for some permutation $\Pi$, then $\Pi^{T} \Pi=I$. Therefore, $\Pi^{-1}(s)=\Pi^{T}(s)$.

The product $B=A \Pi(s)$ is the matrix whose columns are the permuted columns of $A$ with respect to the permutation $\pi(s)$ and $\Pi B$ is the matrix whose rows are the permuted rows of $B$ and also with respect to $\pi$.

In addition, note that (1.1) is equivalent to

$$
a_{j k}=a_{\pi(s)(j), \pi(s)(k)} \quad \text { for } j=1, \ldots, n, k=1, \ldots, n .
$$

\subsection{Some equivariant matrices}

1.2.1. Symmetric Toeplitz matrices. Toeplitz matrices arise in many fields of applications, such as signal processing, coding theory, speech analysis, probability, and statistics.

DEFINITION 1.3. An $n \times n$ symmetric Toeplitz matrix is characterized by the property that it has constant diagonals from top left to the bottom right. The general case is

$$
T_{n}=\left(\begin{array}{ccccc}
a_{11} & a_{12} & \cdots & a_{1 n-1} & a_{1 n} \\
a_{12} & a_{11} & \cdots & a_{1 n-2} & a_{1 n-1} \\
\vdots & \vdots & \ddots & \vdots & \vdots \\
a_{1 n} & a_{1 n-1} & \cdots & a_{12} & a_{11}
\end{array}\right) .
$$

Note that $\pi=(n n-1 n-2 \cdots 321)$ and the corresponding permutation matrix is

$$
\Pi=\left(\begin{array}{ccc}
0 & \cdots & 1 \\
\vdots & & \vdots \\
1 & \cdots & 0
\end{array}\right)
$$

It is clear that (1.3) holds for $T_{n}$. Matrices $T_{n}$ are equivariant matrices with this cyclic group of two elements. In fact, this holds also for any symmetric matrix.

\subsubsection{Equivariant circulant matrices}

DEFINITION 1.4. An $n \times n$ matrix $C_{n}$ is a circulant matrix if it is of the form:

$$
C_{n}=\left(\begin{array}{ccccc}
c_{1} & c_{2} & c_{3} & \cdots & c_{n} \\
c_{n} & c_{1} & c_{2} & \cdots & c_{n-1} \\
& \ddots & \ddots & \ddots & \vdots \\
c_{2} & c_{3} & c_{4} & \cdots & c_{1}
\end{array}\right) .
$$


For the permutation $\pi=\left(\begin{array}{lllll}1 & 2 & 3 & \cdots & n\end{array}\right)$, obviously (1.3) holds and

$$
\Pi=\left(\begin{array}{ccccc}
0 & 0 & \cdots & 0 & 1 \\
1 & 0 & \cdots & 0 & 0 \\
0 & 1 & \cdots & 0 & 0 \\
0 & 0 & \cdots & \vdots & \vdots \\
\vdots & \vdots & & 0 & 0 \\
0 & 0 & \cdots & 1 & 0
\end{array}\right) .
$$

The group here is a special group, the cyclic group of permutations: $\left\{e, \pi, \pi^{2}, \ldots, \pi^{n-1}\right\}$ group $\pi$. Note that $C_{n}$ has the equivariance of the cyclic group $\left\{e, \pi, \pi^{2}, \ldots, \pi^{n-1}\right\}$ of order $n . C_{n}$ commutes with the permutation matrix $\Pi$.

If $\Gamma$ is a finite group, $H$ a finite dimensional vector space, $R: \Gamma \rightarrow L(H)$ a linear representation (i.e., an action) of $\Gamma$ on $H$, and $A: H \rightarrow H$ a linear operator which commutes with $R$ (i.e., is equivariant), then it is well known that $H$ splits into a canonical direct sum

$$
H=\bigoplus_{\mathbf{r}, k} H_{\mathbf{r}, k}
$$

via the projectors

$$
P_{\mathbf{r}, k}=\frac{\operatorname{dim} \mathbf{r}}{|\Gamma|} \sum_{g \in \Gamma} \mathbf{r}\left(g^{-1}\right)[k, k] R(g),
$$

where $\mathbf{r}$ runs through a complete list of irreducible representations of $\Gamma$ and $1 \leq \rho \leq$ dimr. It is well known that the equivariance of $A$ leads to a splitting

$$
A=\bigoplus_{\mathbf{r}, \rho} A_{\mathbf{r}, \rho}
$$

where $A_{\mathbf{r}, \rho}: H_{\mathbf{r}, \rho} \rightarrow H_{\mathbf{r}, \rho}$. This can be exploited to solve linear equations or eigenvalue problems involving $A$. Hence the linear equations or eigenvalue problems are solved over each of the subspaces $H_{\mathbf{r}, \rho}$. We call this approach the symmetry reduction method. In [4] methods of implementing the symmetry reduction method are described in detail.

2. Applications. Equivariant matrices occur in many scientific phenomena of mathematics, physics, engineering, etc. We have chosen statistics to be our target of applications. In this section, we present some applications for equivariant matrices. The first one is the well-known Fourier transformation and the second one is the stationary processes in time series analysis.

2.1. Fourier transforms. Fourier transforms on finite groups have various applications: Fourier transforms on finite cyclic groups are used for fast polynomial and integer multiplication $[18,19]$. The Fourier transforms corresponding to elementary abelian 2-groups are called Walsh-Hadamard transforms. They play an important role in digital image processing and in complexity analysis. Diaconis [12] uses Fourier transforms on symmetric groups for the variance analysis of ranked data. Further 
applications of Fourier transforms on non-abelian groups to problems in combinatorics, theoretical computer science, probability theory and statistics are described by Diaconis in [11].

We have recently had to compute Fourier transforms on the symmetric group $S_{n}$ as part of a statistical analysis of rankings in an election. Here $n$ is the number of candidates, and $f(\pi)$ is the number of voters choosing the rank order $\pi$. Let $\Pi=\rho(\pi)$ be the permutation matrix for $\pi$. The entries of $\Pi$, say $(i, j)$, are 1 in position $(i, j)$ if $\pi(i)=j$. Hence the Fourier transforms

$$
\hat{f}(\rho)=\sum_{\pi} f(\pi) \rho(\pi)
$$

counts how many voters ranked candidate $i$ in position $j$. Diaconis and Rockmore [13] derived fast algorithms for computing $\hat{f}(\rho)$ and developed them for symmetric groups. The Fourier transform(s) is closely related to the generalized Fourier transform used in [4] for the symmetry reduction method.

\subsection{Stationary time series processes}

Definition 2.1. A time series $\left\{X_{t}, t \in Z\right\}$, with index set $Z=\{0, \pm 1, \pm 2, \ldots\}$ is said to be stationary if

(i) $E\left|X_{t}\right|^{2}<\infty$ for all $t \in Z$,

(ii) $E X_{t}=m$ for all $t \in Z$,

(iii) $\gamma_{X}(h)=\operatorname{Cov}\left(X_{t+h}, X_{t}\right)$ for all $t, h \in Z$,

where $E$ is the expected value for the random series $X_{t}$ and "Cov" refers to the covariance function between any two random series defined as follows.

DEFINITION 2.2 (expectation). Let $X$ be a random variable. The expected value or the mean of $X$ denoted by $E X$ is defined by

$$
E X=\int_{0}^{\infty}\left[1-F_{X}(x)\right] d x-\int_{-\infty}^{0} F_{X}(x) d x
$$

where $F_{X}(\cdot)$ is the distribution function of the random variable $X$.

DEFINITION 2.3 (covariance). Let $X$ and $Y$ be any two random variables defined on the same probability space. The covariance of $X$ and $Y$ denoted by $\gamma_{X, Y}(\cdot)$ is defined as

$$
\gamma_{X, Y}(\cdot)=\operatorname{Cov}(X, Y)=E[(X-E X)(Y-E Y)]
$$

Note that this definition is equivalent to saying that for any given time series to be stationary it has to have a finite second moment, the first moment is constant over time and the covariance function depends only on the difference of the time. From this definition we see that the third property implies

$$
\operatorname{Cov}\left(X_{t}, X_{t+h}\right)=\operatorname{Cov}\left(X_{t-h}, X_{t}\right)=\operatorname{Cov}\left(X_{t}, X_{t-h}\right)=\gamma_{X}(h) .
$$


In a matrix format, if we have $x_{1}, x_{2}, \ldots, x_{n}$ observed $n$ time series, then

$$
\Gamma_{n}(h)=\left(\begin{array}{cccc}
\gamma(0) & \gamma(1) & \cdots & \gamma(n-1) \\
\gamma(1) & \gamma(0) & \cdots & \gamma(n-2) \\
\vdots & \vdots & \ddots & \vdots \\
\gamma(n-1) & \gamma(n-2) & \cdots & \gamma(0)
\end{array}\right)
$$

which is an $n \times n$ symmetric Toeplitz matrix as it was defined in (1.4) with permutation $\pi=(n n-1 n-2 \cdots 321)$. It is well known that a covariance matrix must be a nonnegative definite matrix and most cases positive definite. This matrix enters in a linear system as we will see in (2.12) and (2.15).

For more information on stationary time series processes, see any time series book (cf. [7]).

Since $\Gamma_{n}$ has the equivariance property, we take advantage of this property as Allgower and Fässler [3] mentioned in their paper to minimize the cost of computation in solving linear systems of equations in prediction in stationary time series. This should be effective since usually $n$ is very large, 1000, 3000, etc.

For solving a linear system of the form $A x=b$, where $A$ is an $n$-by- $n$ Toeplitz matrix and because $A$ is completely specified by $2 n-1$ numbers, it is desirable to derive an algorithm for solving Toeplitz systems in less than the $O\left(n^{3}\right)$ complexity for Gaussian elimination for a general matrix. In time series analysis algorithms with $O\left(n^{2}\right)$ complexity have been known for some time and are based on the Levinsion recursion formula [7]. More recently, even faster algorithms with $O\left(n \log ^{2} n\right)$ have been proposed [5, 6] but their stability properties are not yet clearly understood [8].

An alternative is to use iterative methods, based on using matrix-vector products of the form $A v$, which can be computed in $O(n \log n)$ complexity via the fast Fourier transform (FFT). To have any chance of beating the direct methods, such iterations must converge very rapidly and this naturally leads to the search for good preconditioners for $A$. Strang [20] proposed using circulant preconditioners, because circulant systems can be solved efficiently by FFT's in $O(n \log n)$ complexity. In particular if $\Gamma_{n}$ in stationary processes is positive definite, then a circulant preconditioner $S$ can be obtained by copying the central diagonals of $\Gamma_{n}$ and "bringing them around" to complete the circulant.

Chan [9] viewed a preconditioner $C$ for a matrix $A$ in solving a linear system $A x=b$ as an approximation to $A$. He derived an optimal circulant preconditioner $C$ in the sense of minimizing $\|C-A\|$.

2.2.1. A distribution problem in spectral analysis. In connection with stationary processes, certain results on quadratic forms can be used to solve a distribution problem in spectral analysis. Let $X_{t}$ be a real and normally distributed stationary process with a discrete time parameter and with an absolutely continuous spectrum. One of the important problems in the theory of time series is to find an estimate $\hat{f}(\lambda)$ for the spectral density $f(\lambda)$, assuming that the process has been observed for $t=1,2, \ldots, n$. The estimate $\hat{f}(\lambda)$ must be a function of $x_{1}, \ldots, x_{n}$, and there are reasons to require that this function should be a nonnegative quadratic form (Grenander and Rosenblatt 1957). 
Thus an estimate of the form

$$
\hat{f}=\mathbf{X}^{T} W \mathbf{X}
$$

is considered, where $\mathbf{X}^{T}=\left(X_{1}, \ldots, X_{n}\right)$ and $W$ is nonnegative. The exact distribution or approximate distribution of $\hat{f}$ is needed. In almost all important cases, $W$ is a Toeplitz matrix. It is well known that $\hat{f}$ can be reduced to the canonical form

$$
\hat{f}=\sum_{j=1}^{r} \lambda_{j} x_{j}^{2},
$$

where $r$ is the rank of $W$ and $\lambda_{j}$ 's are the eigenvalues of $W$ and the $\chi_{j}^{2}$ 's are independent chi-square random variables with one degree of freedom each. After finding the $\lambda_{j}$ 's it becomes easier to find approximation to the distribution of $\hat{f}$, see Alkarni [1].

The following result shows that if $A$ is equivariant, then the quadratic form $Q(\mathbf{x})=$ $\mathbf{x}^{T} A \mathbf{x}$ is invariant with respect to its permutation group.

THEOREM 2.4. Suppose $A$ is real and equivariant matrix with respect to a permutation group $\Gamma$, then the quadratic form $Q(\mathbf{x})=\mathbf{x}^{T} A \mathbf{x}$ is invariant with respect to the permutation matrix $\Gamma$. Moreover, for all $\pi \in \Gamma$ if $(\lambda, \mathbf{x})$ is an eigenvalue-eigenvector pair, then so is $(\lambda, \Pi \mathbf{x})$ and $\lambda$ has multiplicity equal to $\operatorname{dim}(\operatorname{sp}\{\Pi \mathbf{x}: \Pi \in \Gamma\})$.

Proof. Let $\Pi \in \Gamma$, then

$$
Q(\Pi \mathbf{x})=(\Pi \mathbf{x})^{T} A(\Pi \mathbf{x})=\left(\mathbf{x}^{T} \Pi^{T}\right) A(\Pi \mathbf{x})=\mathbf{x}^{T}\left(\Pi^{T} A \Pi\right) \mathbf{x}=\mathbf{x}^{T} A \mathbf{x}=Q(\mathbf{x}) .
$$

Now suppose $A \mathbf{x}=\lambda \mathbf{x}$, where $A$ is equivariant with respect to a permutation group $\Gamma$ then for all $\Pi \in \Gamma$,

$$
A(\Pi \mathbf{x})=\Pi(A \mathbf{x})=\Pi(\lambda \mathbf{x})=\lambda \Pi \mathbf{x}
$$

which implies that if $(\lambda, \mathbf{x})$ is an eigenvalue-eigenvector pair, then $(\lambda, \Pi \mathbf{x})$ is also an eigenvalue-eigenvector pair.

Suppose now that $\Pi \mathbf{x} \neq \alpha \mathbf{x}$ for every scalar $\alpha \neq 0$. Then $\Pi \mathbf{x}$ is another eigenvector, therefore $\lambda$ has a multiplicity equal to $\operatorname{dim}(\operatorname{sp}\{\Pi \mathbf{x}: \Pi \in \Gamma\})$.

We conclude that the equivariance property helps to know the multiplicities of eigenvalues of a matrix and yields corresponding eigenvectors at a low computational cost. This will reduced the cost of computations.

\subsubsection{Prediction of stationary processes}

(1) ONE-STEP PREDICTORS. Let $\left\{X_{t}\right\}$ be a stationary process with mean zero and auto covariance function $\gamma(\cdot)$. Let $H_{n}$ denote the closed linear subspace $\overline{\operatorname{sp}}\left\{X_{1}, \ldots, X_{n}\right\}$, $n \geq 1$, and let $\hat{X}_{n+1}, n \geq 0$, denote the one-step predictors defined by

$$
\hat{X}_{n+1}= \begin{cases}0, & \text { if } n=0, \\ P_{H_{n}} X_{n+1}, & \text { if } n \geq 1,\end{cases}
$$

where $P_{H_{n}} X_{n+1}$ is the projection of $X_{n+1}$ onto the closed linear subspace $H_{n}$. For more on projection theory see, for example, [10]. 
Since $\hat{X}_{n+1} \in H_{n}, n \geq 1$, we can write

$$
\hat{X}_{n+1}=\phi_{n 1} X_{n}+\cdots+\phi_{n n} X_{1}, \quad n \geq 1 .
$$

Using the projection theory, we end up solving the system

$$
\Gamma_{n} \Phi_{n}=\gamma_{n},
$$

where $\Gamma_{n}=[\gamma(i-j)]_{i, j=1, \ldots, n}$ is the covariance matrix in $(2.5), \gamma_{n}=(\gamma(1), \ldots, \gamma(n))^{T}$ and $\Phi_{n}=\left(\phi_{n 1}, \ldots, \phi_{n n}\right)^{T}$. The projection theorem guarantees that equation (2.12) has at least one solution. Although there may be many solutions to (2.12), every one of them, when substituted into (2.11), must give the same predictor $\hat{X}_{n+1}$ since by projection theory $\hat{X}_{n+1}$ is uniquely defined. There is exactly one solution of (2.12) if and only if $\Gamma_{n}$ is nonsingular in which case the solution is

$$
\Phi_{n}=\Gamma_{n}^{-1} \gamma_{n}
$$

It can be shown that if $\gamma(0)>0$ and $\gamma(h) \rightarrow 0$ as $h \rightarrow \infty$, then the covariance matrix $\Gamma_{n}$ is nonsingular for every $n$. For a proof see [7]. Hence our goal is to find a solution of (2.12) if there are more than one solution or to find the inverse $\Gamma_{n}^{-1}$ if $\Gamma_{n}$ is nonsingular. In either case using the equivariant property of $\Gamma_{n}$ will be useful.

(2) THE $h$-STEP PREDICTORS, $h \geq 1$. In the same manner the best linear predictor of $X_{n+h}$ in terms of $X_{1}, \ldots, X_{n}$ for any $h \geq 1$ can be found to be

$$
P_{H_{n}} X_{n+h}=\phi_{n 1}^{(h)} X_{n}+\cdots+\phi_{n n}^{(h)} X_{1}, \quad n, h \geq 1,
$$

where $\Phi_{n}^{(h)}=\left(\phi_{n 1}^{(h)}, \ldots, \phi_{n n}^{(h)}\right)^{T}$ is any solution (it is unique if $\Gamma_{n}$ is nonsingular) of

$$
\Gamma_{n} \Phi_{n}^{(h)}=\gamma_{n}^{(h)},
$$

where $\gamma_{n}^{(h)}=(\gamma(h), \gamma(h+1), \ldots, \gamma(n+h-1))^{T}$ and $\Gamma_{n}$ is as in (2.5).

As was mentioned before, we need to find a solution to the large system in (2.15) or the unique one if $\Gamma_{n}$ is nonsingular.

The use of the equivariance property will be even more effective if we apply it to the prediction equation of a well-known class of stationary time series processes, the autoregressive moving average or ARMA processes. The use of the equivariant property becomes effective because of the structure of $\Gamma_{n}$, the auto covariance function. For the definition of ARMA and their properties we refer the reader to [7]. We only present the structure of the auto covariance function.

If we have an $\operatorname{ARMA}(p, q)$ model, and if $m=\max (p, q)$, then the auto covariance function is given by

$$
\kappa(i, j)= \begin{cases}\sigma^{-2} \gamma_{X}(i-j), & 1 \leq i, j \leq m, \\ \sigma^{-2}\left[\gamma_{X}(i-j)-\sum_{r=1}^{p} \phi_{r} \gamma_{X}(r-|i-j|)\right], & \min (i, j) \leq m<\max (i, j) \leq 2 m, \\ \sum_{r=0}^{q} \theta_{r} \theta_{r+|i-j|}, & \min (i, j)>m, \\ 0, & \text { otherwise. }\end{cases}
$$


This structure leads to an $n \times n$ block diagonal Toeplitz matrix $\Gamma_{n}$ in which case we apply an algorithm based on the equivariance property to solve the system in (2.12) or in (2.15), which is more efficient than the direct computation.

2.2.3. The Yule-Walker equations and parameter estimation for autoregressive processes. Let $\left\{X_{t}\right\}$ be the zero-mean causal autoregressive process,

$$
X_{t}-\phi_{1} X_{t-1}-\cdots-\phi_{p} X_{t-p}=Z_{t}, \quad\left\{Z_{t}\right\} \sim W N\left(0, \sigma^{2}\right) .
$$

Our aim is to find estimators of the coefficient vector $\phi=\left(\phi_{1}, \ldots, \phi_{p}\right)^{T}$ and the white noise variance $\sigma^{2}$ based on the observations $X_{1}, \ldots, X_{n}$. Because of the causality assumption, that is, $X_{t}$ can be written as a linear combination of $Z_{t}$, we end up solving the linear system

$$
\begin{gathered}
\hat{\Gamma}_{p} \hat{\phi}=\hat{\gamma_{p}}, \\
\hat{\sigma^{2}=} \hat{\gamma}(0)-\hat{\phi}^{T} \hat{\gamma}_{p} .
\end{gathered}
$$

Equations (2.18) and (2.19) are known in time series to be the Yule-Walker estimators $\hat{\phi}$ and $\hat{\sigma}^{2}$ of $\phi$ and $\sigma^{2}$.

The linear system in (2.18) is an equivariant system under the permutation $\pi=$ (n $n-1 n-2 \cdots 321)$.

\section{REFERENCES}

[1] S. H. Akarni, On the distribution of quadratic forms and of their ratios, Ph.D. thesis, Department of Statistics, Colorado State University, Ft. Collins, 1997.

[2] E. L. Allgower, K. Böhmer, K. Georg, and R. Miranda, Exploiting symmetry in boundary element methods, SIAM J. Numer. Anal. 29 (1992), no. 2, 534-552. MR 93b:65184. Zbl 754.65089.

[3] E. L. Allgower and A. F. Fässler, Block structure and equivalence of matrices, Aspects of Complex Analysis, Differential Geometry, Mathematical Physics and Applications (St. Konstantin, 1998), World Sci. Publishing, River Edge, NJ, 1999, pp. 19-34. MR 2000i:15001.

[4] E. L. Allgower, K. Georg, R. Miranda, and J. Tausch, Numerical exploitation of equivariance, Z. Angew. Math. Mech. 78 (1998), no. 12, 795-806. MR 99h:65226. Zbl 919.65018.

[5] R. R. Bitmead and B. D. O. Anderson, Asymptotically fast solution of Toeplitz and related systems of linear equations, Linear Algebra Appl. 34 (1980), 103-116. MR 81m:65044. Zbl 458.65018.

[6] R. P. Brent, F. G. Gustavson, and D. Y. Y. Yun, Fast solution of Toeplitz systems of equations and computation of Padé approximants, J. Algorithms 1 (1980), no. 3, 259-295. MR 82d:65033. Zbl 475.65018.

[7] P. J. Brockwell and R. A. Davis, Time Series: Theory and Methods, 2nd ed., Springer Series in Statistics, Springer-Verlag, New York, 1991. MR 92d:62001. Zbl 709.62080.

[8] J. R. Bunch, Stability of methods for solving Toeplitz systems of equations, SIAM J. Sci. Statist. Comput. 6 (1985), no. 2, 349-364. MR 87a:65073. Zbl 569.65019.

[9] T. F. Chan, An optimal circulant preconditioner for Toeplitz systems, SIAM J. Sci. Statist. Comput. 9 (1988), no. 4, 766-771. MR 89e:65046. Zbl 646.65042.

[10] R. Christensen, Plane Answers to Complex Questions. The Theory of Linear Models, Springer Texts in Statistics, Springer-Verlag, New York, Berlin, 1987. MR 88k:62103. Zbl 645.62076.

[11] P. Diaconis, Group Representations in Probability and Statistics, Institute of Mathematical Statistics Lecture Notes-Monograph Series, vol. 11, Institute of Mathematical Statistics, Hayward, CA, 1988. MR 90a:60001. Zbl 695.60012. 
[12] _ A generalization of spectral analysis with application to ranked data, Ann. Statist. 17 (1989), no. 3, 949-979. MR 91a:60025. Zbl 688.62005.

[13] P. Diaconis and D. Rockmore, Efficient computation of the Fourier transform on finite groups, J. Amer. Math. Soc. 3 (1990), no. 2, 297-332. MR 92g:20024. Zbl 709.65125.

[14] A. Fässler and E. Stiefel, Group Theoretical Methods and their Applications. Translated from the German by Baoswan Dzung Wong, Birkhäuser Boston, Inc., Boston, MA, 1992. MR 93a:20023. Zbl 769.20002.

[15] K. Georg and L. Tausch, User's guide for package to solve equivariant linear systems, Tech. report, Colorado State University, 1995.

[16] M. Golubitsky and D. G. Schaeffer, Singularities and Groups in Bifurcation Theory. Vol. I, Applied Mathematical Sciences, vol. 51, Springer-Verlag, New York, Berlin, 1985. MR 86e:58014. Zbl 607.35004.

[17] M. Golubitsky, I. Stewart, and D. G. Schaeffer, Singularities and Groups in Bifurcation Theory. Vol. II, Applied Mathematical Sciences, vol. 69, Springer-Verlag, New York, Berlin, 1988. MR 89m:58038. Zbl 691.58003.

[18] J. D. Lipson, Elements of Algebra and Algebraic Computing, Addison-Wesley Publishing Co., Reading, Mass., 1981. MR 83f:00005. Zbl 467.12001.

[19] A. Schönhage and V. Strassen, Schnelle Multiplikation grosser Zahlen [Speedy multiplication of great numbers], Computing (Arch. Elektron. Rechnen) 7 (1971), 281-292 (German). MR 45\#1431. Zbl 223.68007.

[20] G. Strang, A proposal for Toeplitz matrix calculations, Stud. Appl. Math. 74 (1986), 171176. Zbl 621.65025.

[21] A. Vanderbauwhede, Local Bifurcation and Symmetry, Research Notes in Mathematics, vol. 75, Pitman (Advanced Publishing Program), Boston, Mass., London, 1982. MR 85f:58026. Zbl 539.58022.

S. H. ALKarni: Department of Statistics, King SAUd University, P.O. Box 2459, RiyadH 11451, SAUDI ARABIA

E-mail address: sa7karni@ksu.edu.sa 


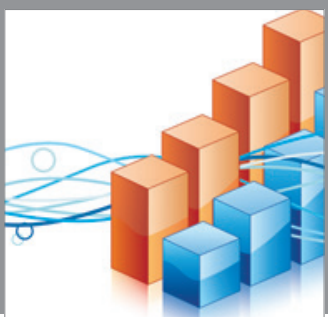

Advances in

Operations Research

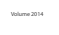

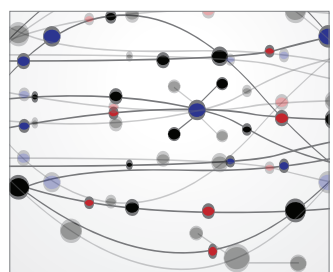

\section{The Scientific} World Journal
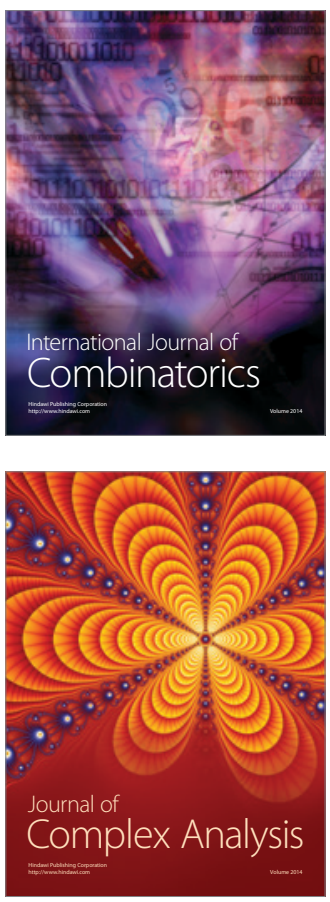

International Journal of

Mathematics and

Mathematical

Sciences
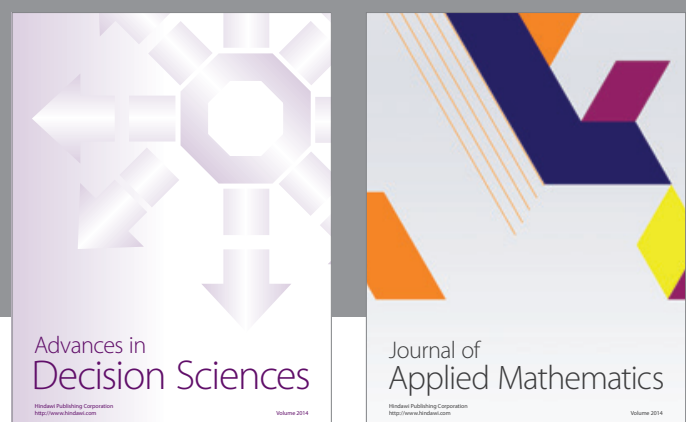

Journal of

Applied Mathematics
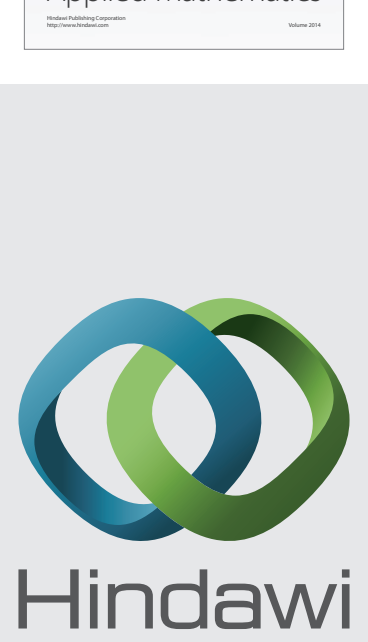

Submit your manuscripts at http://www.hindawi.com
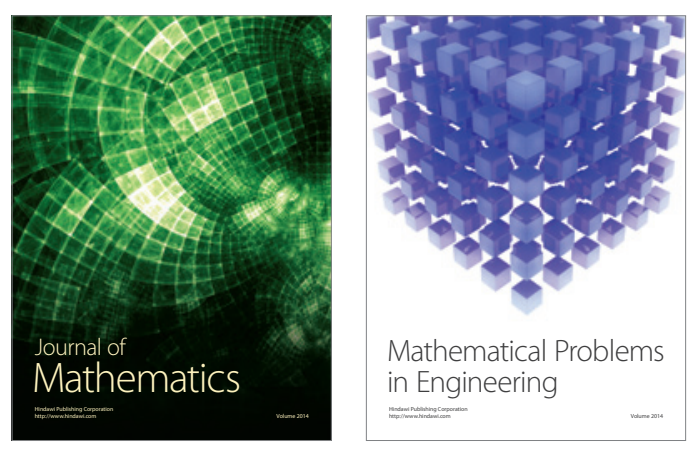

Mathematical Problems in Engineering
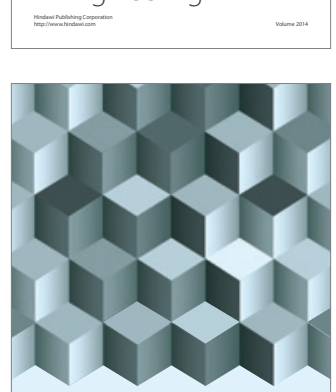

Journal of

Function Spaces
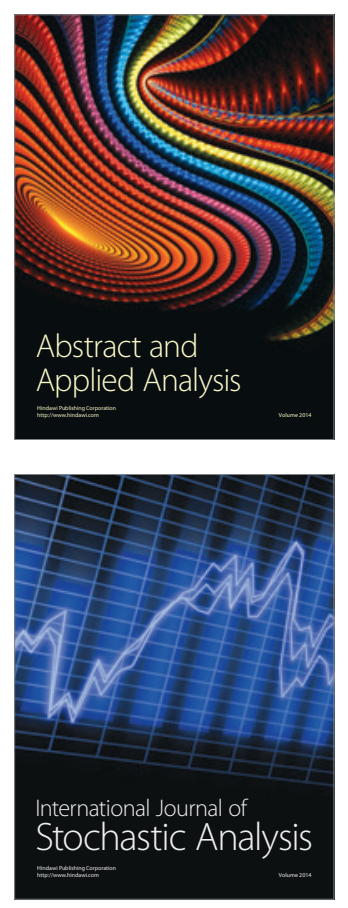

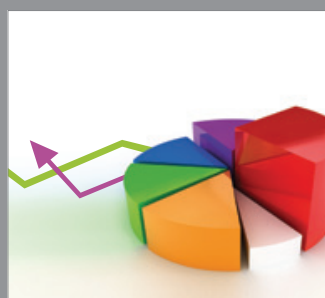

ournal of

Probability and Statistics

Promensencen
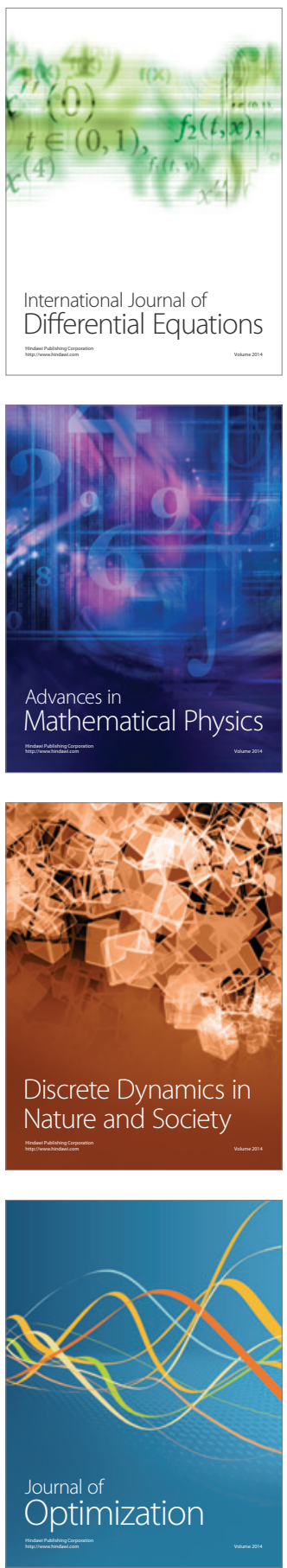